\title{
Global higher integrability for very weak solutions to nonlinear subelliptic equations
}

\section{Guangwei Du and Junqiang Han*}

"Correspondence: southhan@163.com Department of Applied Mathematics, Northwestern Polytechnical University, Xi'an, Shaanxi, 710129, P.R. China

\begin{abstract}
In this paper we consider the following nonlinear subelliptic Dirichlet problem:

$$
\left\{\begin{array}{l}
X^{*} A(x, u, X u)+B(x, u, X u)=0, \quad x \in \Omega, \\
u-u_{0} \in W_{X, 0}^{1, r}(\Omega),
\end{array}\right.
$$

where $X=\left\{X_{1}, \ldots, X_{m}\right\}$ is a system of smooth vector fields defined in $\mathbf{R}^{n}$ with globally Lipschitz coefficients satisfying Hörmander's condition, and we prove the global higher integrability for the very weak solutions.
\end{abstract}

MSC: Primary 35H20; secondary 35J60

Keywords: nonlinear subelliptic equations; very weak solutions; global higher integrability

\section{Introduction and main result}

The theory of very weak solutions was introduced in the work of Iwaniec and Sbordone [1]. Iwaniec and Sbordone realized that the usual Sobolev assumption for weak solutions to $p$-harmonic equation can be relaxed to a slightly weaker Sobolev space and proved that very weak solutions are actually classical weak solutions by using the nonlinear Hodge decomposition to construct suitable test functions. Based on Whitney's extension theorem and theory of $A_{p}$ weights, Lewis [2] showed a completely different proof and obtained the same result to certain elliptic systems. After [1] and [2], many authors have devoted their energy to the study of the regularity of such solutions; see for example [3-5] and the references therein. We mention here that Xie and Fang [5] obtained the global higher integrability for very weak solutions to a class of nonlinear elliptic systems with Lipschitz boundary condition by using Hodge decomposition to construct a suitable test function. Recently the authors in [6] proved the global regularity result for a second-order degenerate elliptic systems of $p$-Laplacian type in the Euclidean setting.

In 2005, Zatorska-Goldstein [7] showed the local higher integrability of very weak solutions to the nonlinear subelliptic equations

$$
X^{*} A(x, u, X u)+B(x, u, X u)=0, \quad x \in \Omega
$$

\section{Springer}


where $\Omega \subset \mathbf{R}^{n}$ is a bounded domain and $X=\left\{X_{1}, \ldots, X_{m}\right\}(m \leq n)$ is a system of smooth vector fields in $\mathbf{R}^{n}$ with globally Lipschitz coefficients satisfying the Hörmander's condition and $X^{*}=\left(X_{1}^{*}, \ldots, X_{m}^{*}\right)$ is a family of operators formal adjoint to $X_{j}$ in $L^{2}$.

In this work we are concerned with the boundary value problem for (1.1) with the boundary condition $u-u_{0} \in W_{X, 0}^{1, r}(\Omega)$, i.e.,

$$
\left\{\begin{array}{l}
X^{*} A(x, u, X u)+B(x, u, X u)=0, \quad x \in \Omega, \\
u-u_{0} \in W_{X, 0}^{1, r}(\Omega)
\end{array}\right.
$$

and establish the global higher integrability for very weak solutions. We assume that the functions $A=\left(A_{1}, \ldots, A_{m}\right): \mathbf{R}^{n} \times \mathbf{R} \times \mathbf{R}^{m} \rightarrow \mathbf{R}^{m}$ and $B: \mathbf{R}^{n} \times \mathbf{R} \times \mathbf{R}^{m} \rightarrow \mathbf{R}$ are both Carathéodory functions satisfying

$$
\begin{aligned}
& |A(x, u, \xi)| \leq \alpha\left(|u|^{p-1}+|\xi|^{p-1}\right), \\
& |B(x, u, \xi)| \leq \alpha\left(|u|^{p-1}+|\xi|^{p-1}\right), \\
& \left\langle A(x, u, \xi)-A(x, v, \zeta), \xi-\zeta|\geq \beta| \xi-\left.\zeta\right|^{2}(|\xi|+|\zeta|)^{p-2},\right.
\end{aligned}
$$

for a.e. $x \in \mathbf{R}^{n}, u \in \mathbf{R}$ and $\xi \in \mathbf{R}^{m}$. Here $p \geq 2, \alpha, \beta$ are positive constants.

A function $u \in W_{X}^{1, r}(\Omega)(r<p)$ is called a very weak solution to (1.1) if

$$
\int_{\Omega} A(x, u, X u) \cdot X \varphi d x+\int_{\Omega} B(x, u, X u) \varphi d x=0
$$

holds for all $\varphi \in C_{0}^{\infty}(\Omega)$.

In the above definition, the very weak means the integrable exponent is strictly lower than the natural exponent $p$ and if $r=p$, this is the classical definition of weak solution to $(1.1)$.

To get our result, some regularity assumption introduced in [8] should be imposed on $\Omega$. Let us first recall the notion of uniform $(X, p)$-fatness which can be found in [9]: A set $E \subset \mathbf{R}^{n}$ is called uniformly $(X, p)$-fat if there exist constants $C_{0}, R_{0}>0$ such that

$$
\operatorname{cap}_{p}(E \cap \bar{B}(x, R), B(x, 2 R)) \geq C_{0} \operatorname{cap}_{p}(\bar{B}(x, R), B(x, 2 R))
$$

for all $x \in \partial E$ and $0<R<R_{0}$, where cap $p$ is the variational $p$-capacity defined in Section 2 .

We consider the following hypotheses on $\Omega$ :

$\left(H_{1}\right)$ there exists a constant $C_{1} \geq 1$ such that, for all $x \in \Omega$,

$$
\left|B_{\rho(x)}\right| \leq C_{1}\left|B_{\rho(x)} \cap\left(\mathbf{R}^{n} \backslash \Omega\right)\right|,
$$

where $\rho(x)=2 \operatorname{dist}\left(x, \mathbf{R}^{n} \backslash \Omega\right)$;

$\left(H_{2}\right)$ the complement $\mathbf{R}^{n} \backslash \Omega$ of $\Omega$ is uniformly $(X, p)$-fat.

Under the hypotheses stated above, we prove the following.

Theorem 1.1 Assume that $u_{0} \in W_{X}^{1, s}(\Omega), s>p$. Then there exists $a \delta>0$ such that if $u \in$ $W_{X}^{1, p-\delta}(\Omega)$ is a very weak solution to the Dirichlet problem $(1.2)$, we have $u \in W_{X}^{1, p+\tilde{\delta}}(\Omega)$ for some $\tilde{\delta}>0$. 
The key technical tool in proving Theorem 1.1 is a Sobolev type inequality with a capacity term. With it we can prove a reverse Hölder inequality for the generalized gradient $X u$ of a very weak solution, which allows us to get the global higher integrability of $X u$. This paper is organized as follows. In Section 2 we collect some known results on CarnotCarathéodory spaces and prove a Sobolev type inequality characterized by capacity. Section 3 is devoted to the proof of Theorem 1.1.

\section{Some known results and a Sobolev type inequality}

Let $\left\{X_{1}, \ldots, X_{m}\right\}$ be a system of $C^{\infty}$-smooth vector fields in $\mathbf{R}^{n}(n \geq 3)$ satisfying Hörmander's condition (see [10]):

$$
\operatorname{rank}\left(\operatorname{Lie}\left\{X_{1}, \ldots, X_{m}\right\}\right)=n
$$

The generalized gradient is denoted by $X u=\left(X_{1} u, \ldots, X_{m} u\right)$ and its length is given by

$$
|X u(x)|=\left(\sum_{j=1}^{m}\left|X_{j} u(x)\right|^{2}\right)^{\frac{1}{2}} .
$$

An absolutely continuous curve $\gamma:[a, b] \rightarrow \mathbf{R}^{n}$ is said to be admissible with respect to the system $\left\{X_{1}, \ldots, X_{m}\right\}$, if there exist functions $c_{i}(t), a \leq t \leq b$, satisfying

$$
\sum_{i=1}^{m} c_{i}(t)^{2} \leq 1 \quad \text { and } \quad \gamma^{\prime}(t)=\sum_{i=1}^{m} c_{i}(t) X_{i}(\gamma(t)) .
$$

The Carnot-Carathéodory distance $d(x, y)$ generated by $\left\{X_{1}, \ldots, X_{m}\right\}$ is defined as the infimum of those $T>0$ for which there exists an admissible path $\gamma:[0, T] \rightarrow \mathbf{R}^{n}$ with $\gamma(0)=x$, $\gamma(T)=y$.

By the accessibility theorem of Chow [11], the distance $d$ is a metric and therefore $\left(\mathbf{R}^{n}, d\right)$ is a metric space which is called the Carnot-Carathéodory space associated with the system $\left\{X_{1}, \ldots, X_{m}\right\}$. The ball is denoted by

$$
B\left(x_{0}, R\right)=\left\{x \in \mathbf{R}^{n}: d\left(x, x_{0}\right)<R\right\} .
$$

For $\sigma>0$ and $B=B\left(x_{0}, R\right)$, we will write $\sigma B$ to indicate $B\left(x_{0}, \sigma R\right)$ and diam $\Omega$ the diameter of $\Omega$ with respect to $d$.

It was proved in [12] that the identity map is a homeomorphism of $\left(\mathbf{R}^{n}, d\right)$ into $\mathbf{R}^{n}$ with the usual Euclidean metric, and every set which is bounded with respect to the Euclidean metric is also bounded with respect to $d$. Moreover, by a result of Garofalo and Nhieu [13], Proposition 2.11, if the given vector fields have globally Lipschitz coefficients in addition, then a subset of $\mathbf{R}^{n}$ is bounded with respect to $d$ if and only if it is bounded with respect to the Euclidean metric.

Hereafter we assume that the vector fields $X_{1}, \ldots, X_{m}$ satisfy the Hörmander condition and have globally Lipschitz coefficients.

Lemma $2.1([12,14])$ For every bounded open set $\Omega \subset \mathbf{R}^{n}$ there exists $C_{d} \geq 1$ such that

$$
|B(x, 2 R)| \leq C_{d}|B(x, R)|
$$

for any $x \in \Omega$ and $0<R \leq 5 \operatorname{diam} \Omega$. 
Here, $|B(x, R)|$ denotes the Lebesgue measure of $B(x, R)$. The best constant $C_{d}$ in (2.1) is called the doubling constant, the measure such that (2.1) holds is called a doubling measure and the homogeneous dimension relative to $\Omega$ is $Q=\log _{2} C_{d}$.

Given $1 \leq p<\infty$, we define the Sobolev space $W_{X}^{1, p}(\Omega)$ by

$$
W_{X}^{1, p}(\Omega)=\left\{u \in L^{p}(\Omega): X_{j} u \in L^{p}(\Omega), j=1,2, \ldots, m\right\}
$$

endowed with the norm

$$
\|u\|_{W_{X}^{1, p}(\Omega)}=\|u\|_{L^{p}(\Omega)}+\|X u\|_{L^{p}(\Omega)} .
$$

Here, $X_{j} u$ is the distributional derivative of $u \in L_{\text {loc }}^{1}(\Omega)$ given by the identity

$$
\left\langle X_{j} u, \varphi\right\rangle=\int_{\Omega} u X_{j}^{*} \varphi d x, \quad \varphi \in C_{0}^{\infty}(\Omega) .
$$

The space $W_{X}^{1, p}(\Omega)$ is a Banach space which admits $C^{\infty}(\Omega) \cap W_{X}^{1, p}(\Omega)$ as its dense subset. The completion of $C_{0}^{\infty}(\Omega)$ under the norm $\|\cdot\|_{W_{X}^{1, p}(\Omega)}$ is denoted by $W_{X, 0}^{1, p}(\Omega)$. The following Sobolev-Poincaré inequalities can be found in [14] and [15]:

Lemma 2.2 Let $Q$ be the homogeneous dimension relative to $\Omega, B=B\left(x_{0}, R\right) \subset \Omega, 0<R<$ $\operatorname{diam} \Omega, 1 \leq p<\infty$. There exists a constant $C>0$ such that, for every $u \in W_{X}^{1, p}(B)$,

$$
\left(f_{B}\left|u-u_{B}\right|^{\kappa p} d x\right)^{\frac{1}{\kappa p}} \leq C R\left(f_{B}|X u|^{p} d x\right)^{\frac{1}{p}}
$$

where $u_{B}=f_{B} u d x=\frac{1}{|B|} \int_{B} u d x$, and $1 \leq \kappa \leq Q /(Q-p)$, if $1 \leq p<Q ; 1 \leq \kappa<\infty$, if $p \geq Q$. Moreover, for any $u \in W_{X, 0}^{1, p}(B)$,

$$
\left(f_{B}|u|^{\kappa p} d x\right)^{\frac{1}{\kappa p}} \leq C R\left(f_{B}|X u|^{p} d x\right)^{\frac{1}{p}}
$$

Next we recall a Gehring lemma on the metric measure space $(Y, d, \mu)$, where $d$ is a metric and $\mu$ is a doubling measure.

Lemma 2.3 ([7]) Let $q \in\left[q_{0}, 2 Q\right], q_{0}>1$ is fixed. Assume that functions $f, g$ are nonnegative and $g \in L_{\mathrm{loc}}^{q}(Y, \mu), f \in L_{\mathrm{loc}}^{r_{0}}(Y, \mu)$, for some $r_{0}>q$. If there exist constants $b>1$ and $\theta$ such that for every ball $B \subset \sigma B \subset Y$ the following inequality holds:

$$
f_{B} g^{q} d \mu \leq b\left[\left(f_{\sigma B} g d \mu\right)^{q}+f_{\sigma B} f^{q} d \mu\right]+\theta f_{\sigma B} g^{q} d \mu
$$

then there exist nonnegative constants $\theta_{0}=\theta_{0}\left(q_{0}, Q, C_{d}, \sigma\right)$ and $\varepsilon_{0}=\varepsilon_{0}\left(b, q_{0}, Q, C_{d}, \sigma\right)$ such that if $0<\theta<\theta_{0}$ then $g \in L_{\mathrm{loc}}^{p}(Y, \mu)$ for $p \in\left[q, q+\varepsilon_{0}\right)$.

For the Hardy-Littlewood maximal functions

$$
M f(x)=\sup _{R>0} \frac{1}{|B(x, R)|} \int_{B(x, R)}|f(y)| d y
$$


and

$$
M_{\Omega} f(x)=\sup _{R>0} \frac{1}{|B(x, R)|} \int_{B(x, R) \cap \Omega}|f(y)| d y
$$

we will use the following properties proved in [14] and [15].

Lemma 2.4 If $f \in L^{p}(\Omega), 1<p \leq \infty$, then $M_{\Omega} f \in L^{p}(\Omega)$ and there exists a constant $C=$ $C\left(C_{d}, p\right)>0$ such that

$$
\left\|M_{\Omega} f\right\|_{L^{p}(\Omega)} \leq C\|f\|_{L^{p}(\Omega)}
$$

Lemma 2.5 If $u \in W_{X, l o c}^{1, p}(\Omega), 1<p<\infty$, then there exists $C>0$ such that, for a.e. $x, y \in \Omega$,

$$
|u(x)-u(y)| \leq C d(x, y)\left(M_{\Omega}|X u|(x)+M_{\Omega}|X u|(y)\right)
$$

Moreover, for any $B=B\left(x_{0}, R\right) \subset \Omega$ and $u \in W_{X}^{1, p}(B)$, we have

$$
\left|u(x)-u_{B}\right| \leq C R M_{B}|X u|(x), \quad \text { a.e. } x \in B \text {. }
$$

It is worth noting that from Lemma 2.5 and Lemma 2.2 we can infer that, for a.e. $x \in B$ and $u \in W_{X, 0}^{1, p}(B)$,

$$
|u(x)| \leq C R M_{B}|X u|(x) .
$$

Let $\omega(x) \geq 0$ be a locally integrable function, we say that $\omega \in A_{p}, 1<p<\infty$, if there exists some positive constant $A$ such that

$$
\sup _{B \subset \mathbf{R}^{n}}\left(f_{B} \omega d x\right)\left(f_{B} \omega^{\frac{1}{1-p}} d x\right)^{p-1} \leq A<\infty .
$$

Lemma 2.6 Assume $\omega \in L_{\mathrm{loc}}^{1}\left(\mathbf{R}^{n}\right)$ is nonnegative and $1<p<\infty$. Then $\omega \in A_{p}$ if and only if there exists a constant $C>0$ such that

$$
\int_{\mathbf{R}^{n}}|M f|^{p} \omega d x \leq C \int_{\mathbf{R}^{n}}|f|^{p} \omega d x
$$

for all $f \in L^{p}(\omega(x) d x)$.

The $(X, p)$-capacity of a compact set $K \subset \Omega$ in $\Omega$ is defined by

$$
\operatorname{cap}_{p}(K, \Omega)=\inf \left\{\int_{\Omega}|X u|^{p} d x: u \in C_{0}^{\infty}(\Omega), u=1 \text { on } K\right\}
$$

and for an arbitrary set $E \subset \Omega$, the $(X, p)$-capacity of $E$ is

$$
\operatorname{cap}_{p}(E, \Omega)=\inf _{\substack{G \subset \Omega \text { open } \\ E \subset G}} \sup _{\substack{K \subset G \\ K \text { compact }}} \operatorname{cap}_{p}(K, \Omega)
$$


We will use the following two-sided estimate of $(X, p)$-capacity in [16]: For $x \in \Omega$ and $0<R<\operatorname{diam} \Omega$, there exist $C_{1}, C_{2}>0$ such that

$$
C_{1} \frac{|B(x, R)|}{R^{p}} \leq \operatorname{cap}_{p}(\bar{B}(x, R), B(x, 2 R)) \leq C_{2} \frac{|B(x, R)|}{R^{p}} .
$$

Lemma 2.7 ([9]) If $\mathbf{R}^{n} \backslash \Omega$ is uniformly $(X, p)$-fat, then there exists $1<q<p$ such that $\mathbf{R}^{n} \backslash \Omega$ is also uniformly $(X, q)$-fat.

The uniform $(X, q)$-fatness also implies uniform $(X, p)$-fatness for all $p \geq q$, which is a simple consequence of Hölder's and Young's inequality.

At the end of this section we prove a Sobolev type inequality characterized by capacity. A similar inequality in the Euclidean setting can be found in [8].

Lemma 2.8 Let $\Omega \subset \mathbf{R}^{n}$ be a bounded open set with the homogeneous dimension $Q, 1<$ $q<\infty$ and $0<R<\operatorname{diam} \Omega$. For any $x \in \Omega$, denote $B=B(x, R)$ and $N(\varphi)=\{y \in \bar{B}: \varphi(y)=0\}$. Then there exists a constant $C=C(Q, q)>0$ such that, for all $\varphi \in C^{\infty}(2 B) \cap W_{X}^{1, q}(2 B)$,

$$
\left(f_{2 B}|\varphi|^{\kappa q} d x\right)^{\frac{1}{\kappa q}} \leq C\left(\frac{1}{\operatorname{cap}_{q}(N(\varphi), 2 B)} \int_{2 B}|X \varphi|^{q} d x\right)^{\frac{1}{q}},
$$

where $1 \leq \kappa \leq Q /(Q-q)$ if $1 \leq q<Q$ and $1 \leq \kappa<\infty$ if $q \geq Q$.

Proof We always assume $\varphi_{2 B} \neq 0$; otherwise, (2.5) follows immediately from Lemma 2.2 and (2.4). Let $\eta \in C_{0}^{\infty}(2 B), 0 \leq \eta \leq 1$ such that $\eta=1$ on $\bar{B}$ and $|X \eta| \leq \frac{c}{R}$. Denoting $v=$ $\eta\left(\varphi_{2 B}-\varphi\right) / \varphi_{2 B}$, then $v \in C_{0}^{\infty}(2 B)$ and $v=1$ in $N(\varphi)$. It follows from Lemma 2.2 that

$$
\begin{aligned}
\operatorname{cap}_{q}(N(\varphi), 2 B) & \leq \int_{2 B}|X v|^{q} d x \\
& \leq\left|\varphi_{2 B}\right|^{-q} \int_{2 B}|X \eta|^{q}\left|\varphi-\varphi_{2 B}\right|^{q} d x+\left|\varphi_{2 B}\right|^{-q} \int_{2 B}|X \varphi|^{q} d x \\
& \leq C\left|\varphi_{2 B}\right|^{-q} \int_{2 B}|X \varphi|^{q} d x,
\end{aligned}
$$

and then

$$
\left|\varphi_{2 B}\right| \leq C\left(\frac{1}{\operatorname{cap}_{q}(N(\varphi), 2 B)} \int_{2 B}|X \varphi|^{q} d x\right)^{\frac{1}{q}} .
$$

Then Lemma 2.2 and (2.6) lead to

$$
\begin{aligned}
\left(f_{2 B}|\varphi|^{\kappa q} d x\right)^{\frac{1}{\kappa q}} & \leq\left(f_{2 B}\left|\varphi-\varphi_{2 B}\right|^{\kappa q} d x\right)^{\frac{1}{\kappa q}}+\left|\varphi_{2 B}\right| \\
& \leq C R\left(f_{2 B}|X \varphi|^{q} d x\right)^{\frac{1}{q}}+C\left(\frac{1}{\operatorname{cap}_{q}(N(\varphi), 2 B)} \int_{2 B}|X \varphi|^{q} d x\right)^{\frac{1}{q}} \\
& \leq C\left(\frac{1}{\operatorname{cap}_{q}(N(\varphi), 2 B)} \int_{2 B}|X \varphi|^{q} d x\right)^{\frac{1}{q}},
\end{aligned}
$$


where in the last step we used the estimate

$$
\operatorname{cap}_{q}(N(\varphi), 2 B) \leq \operatorname{cap}_{q}(\bar{B}, 2 B) \leq C|B| R^{-q}
$$

The proof is complete.

\section{Proof of Theorem 1.1}

Assume that the function $u \in W_{X}^{1, p-\delta}(\Omega)\left(\delta<\frac{1}{2}\right)$ is a very weak solution to the Dirichlet problem (1.2). Choose a ball $B_{0}$ such that $\bar{\Omega} \subset \frac{1}{2} B_{0}$ and let $B$ be a ball of radius $R$ with $3 B \subset B_{0}$ for fixed $0<R<1$. There are two cases: (i) $3 B \subset \Omega$ or (ii) $3 B \backslash \Omega \neq \emptyset$. In the case (i), the following estimate has been proved in [7]:

$$
f_{\frac{B}{2}}|X u|^{p-\delta} d x \leq \theta f_{3 B}|X u|^{p-\delta} d x+b\left[f_{3 B}|u|^{p-\delta}+\left(f_{3 B}|X u|^{t} d x\right)^{\frac{p-\delta}{t}}\right]
$$

where $\theta$ small enough, $b>1, \max \left\{1,(p-\delta)_{*}\right\}<t<p-\delta$.

When $3 B \backslash \Omega \neq \emptyset$, a similar inequality (see (3.31) below) will be achieved.

Step 1 . Let $\eta$ be a smooth cut-off function on $2 B$, i.e. $\eta \in C_{0}^{\infty}(2 B)$ such that

$$
0 \leq \eta \leq 1, \quad \eta=1 \quad \text { on } B \text { and } \quad|X \eta| \leq c / R \text {. }
$$

Define $\hat{u}=\eta\left(u-u_{0}\right)$ and

$$
E_{\mu}=\left\{x \in \mathbf{R}^{n}: M|X \hat{u}|(x) \leq \mu\right\}, \quad \text { for } \mu>0 .
$$

We conclude from Lemma 2.5 and the assumption $\left(H_{1}\right)$ that $\hat{u}$ is Lipschitz continuous on $E_{\mu} \cup\left(\mathbf{R}^{n} \backslash \Omega\right)$.

Indeed, if $x, y \in E_{\mu} \cap \Omega$, then Lemma 2.5 implies $|\hat{u}(x)-\hat{u}(y)| \leq c \mu d(x, y)$; if $x, y \in \mathbf{R}^{n} \backslash \Omega$, then $\hat{u}(x)=\hat{u}(y)=0$. We set $B_{\rho_{x}}=B\left(x, \rho_{x}\right)$ with $\rho_{x}=2 \operatorname{dist}\left(x, \mathbf{R}^{n} \backslash \Omega\right)$ for the case $x \in E_{\mu} \cap \Omega$ and $y \in \mathbf{R}^{n} \backslash \Omega$. Since $\hat{u}$ is zero on $\mathbf{R}^{n} \backslash \Omega$, it follows that

$$
\begin{aligned}
\int_{B_{\rho_{x}} \cap\left(\mathbf{R}^{n} \backslash \Omega\right)}\left|\hat{u}-\hat{u}_{B_{\rho_{x}}}\right| d z & =\int_{B_{\rho_{x}} \cap\left(\mathbf{R}^{n} \backslash \Omega\right)}\left|\hat{u}_{B_{\rho_{x}}}\right| d z \\
& =\left|B_{\rho_{x}} \cap\left(\mathbf{R}^{n} \backslash \Omega\right)\right|\left|\hat{u}_{B_{\rho_{x}}}\right|
\end{aligned}
$$

and then, from assumption $\left(H_{1}\right)$ and Lemma 2.2,

$$
\begin{aligned}
\left|\hat{u}_{B_{\rho_{x}}}\right| & \leq C_{1} \frac{\left|B_{\rho_{x}} \cap\left(\mathbf{R}^{n} \backslash \Omega\right)\right|}{\left|B_{\rho_{x}}\right|}\left|\hat{u}_{B_{\rho_{x}}}\right| \\
& =\frac{C_{1}}{\left|B_{\rho_{x}}\right|} \int_{B_{\rho_{x}} \cap\left(\mathbf{R}^{n} \backslash \Omega\right)}\left|\hat{u}-\hat{u}_{B_{\rho_{x}}}\right| d z \\
& \leq C_{1} f_{B_{\rho_{x}}}\left|\hat{u}-\hat{u}_{B_{\rho_{x}}}\right| d z \leq c C_{1} \rho_{x} f_{B_{\rho_{x}}}|X \hat{u}| d z \\
& \leq c C_{1} \rho_{x} M|X \hat{u}|(x) \leq c C_{1} \mu \rho_{x} .
\end{aligned}
$$


Therefore, we have by (2.2) and (3.2)

$$
\begin{aligned}
|\hat{u}(x)-\hat{u}(y)| & =|\hat{u}(x)| \\
& \leq\left|\hat{u}(x)-\hat{u}_{B_{\rho_{x}}}\right|+\left|\hat{u}_{B_{\rho_{x}}}\right| \\
& \leq c \rho_{x} M|X \hat{u}|(x)+c C_{1} \mu \rho_{x} \\
& \leq c C_{1} \mu \rho_{x} \\
& \leq c C_{1} \mu d(x, y) .
\end{aligned}
$$

It follows that $\hat{u}$ is a Lipschitz function on $E_{\mu} \cup\left(\mathbf{R}^{n} \backslash \Omega\right)$ with the Lipschitz constant $c C_{1} \mu$. As in [7], we can use the Kirszbraun theorem (see e.g. [17]) to extend $\hat{u}$ to a Lipschitz function $v_{\mu}$ defined on $\mathbf{R}^{n}$ with the same Lipschitz constant. Moreover, there exists $\mu_{0}$ such that, for every $\mu \geq \mu_{0}$, supp $v_{\mu} \subset 3 B \cap \Omega$.

In fact, let $D=2 B \cap \Omega$ and $x \in \mathbf{R}^{n} \backslash(3 B \cap \Omega)$, we have by Lemma 2.1 that

$$
M|X \hat{u}|(x)=\sup _{B^{\prime} \ni x, B^{\prime} \cap 2 B \neq \emptyset \emptyset} f_{B^{\prime}}|X \hat{u}|(y) d y \leq \frac{C_{d}}{|2 B|} \int_{D}|X \hat{u}|(y) d y,
$$

where $\left|B^{\prime}\right|>|B|, C_{d}$ is the doubling constant. Setting

$$
\mu_{0}=\frac{C_{d}}{|2 B|} \int_{D}|X \hat{u}|(y) d y
$$

then $M|X \hat{u}|(x) \leq \mu, \mu \geq \mu_{0}$, which implies $v_{\mu}(x)=\hat{u}(x)=0$ for $x \in \mathbf{R}^{n} \backslash(3 B \cap \Omega)$. So we can take the function $v_{\mu}$ as a test function in (1.6).

Let $\mu \geq \mu_{0}$ and take $v_{\mu}$ as a test function in (1.6) to have

$$
\int_{3 B \cap \Omega} A(x, u, X u) \cdot X v_{\mu} d x+\int_{3 B \cap \Omega} B(x, u, X u) v_{\mu} d x=0
$$

Noting that $v_{\mu}=\hat{u}$ on $(3 B \cap \Omega) \cap E_{\mu}$ and that supp $\hat{u} \subset D$, we have by the structure conditions on $A(x, u, \xi)$ and $B(x, u, \xi)$

$$
\begin{aligned}
& \int_{D \cap E_{\mu}} A(x, u, X u) \cdot X \hat{u} d x+\int_{D \cap E_{\mu}} B(x, u, X u) \hat{u} d x \\
& \quad \leq \int_{(3 B \cap \Omega) \backslash E_{\mu}}|A(x, u, X u)|\left|X v_{\mu}\right| d x+\int_{(3 B \cap \Omega) \backslash E_{\mu}}|B(x, u, X u)|\left|v_{\mu}\right| d x \\
& \quad \leq c \mu \int_{(3 B \cap \Omega) \backslash E_{\mu}}\left(|u|^{p-1}+|X u|^{p-1}\right) d x,
\end{aligned}
$$

where in the last inequality we use the fact that $\left|X v_{\mu}\right| \leq c \mu,\left|v_{\mu}\right| \leq c R \mu$ (see [7]). Multiplying both sides of (3.3) by $\mu^{-(1+\delta)}$ and integrating over $\left(\mu_{0}, \infty\right)$, we get

$$
\begin{aligned}
L & :=\int_{\mu_{0}}^{\infty} \int_{D \cap E_{\mu}} \mu^{-(1+\delta)}(A(x, u, X u) \cdot X \hat{u}+B(x, u, X u) \hat{u}) d x d \mu \\
& \leq c \int_{\mu_{0}}^{\infty} \int_{(3 B \cap \Omega) \backslash E_{\mu}} \mu^{-\delta}\left(|u|^{p-1}+|X u|^{p-1}\right) d x d \mu:=P .
\end{aligned}
$$


Interchanging the order of integration and applying (3.2), we have

$$
\begin{aligned}
P & =c \int_{3 B} \int_{\mu_{0}}^{M|X \hat{u}|} \mu^{-\delta}\left(|u|^{p-1}+|X u|^{p-1}\right) d \mu d x \\
& \leq \frac{c}{1-\delta} \int_{(3 B \cap \Omega) \backslash E_{\mu_{0}}}(M|X \hat{u}|)^{1-\delta}\left(|u|^{p-1}+|X u|^{p-1}\right) d x \\
& \leq c \int_{3 B \cap \Omega}\left(|u|^{p-\delta}+|X u|^{p-\delta}\right) d x+c \int_{3 B \cap \Omega}(M|X \hat{u}|)^{p-\delta} d x .
\end{aligned}
$$

Using Lemma 2.4 and Lemma 2.8, we have

$$
\begin{aligned}
& c \int_{3 B \cap \Omega}(M|X \hat{u}|)^{p-\delta} d x \\
& \quad \leq c \int_{D}|X \hat{u}|^{p-\delta} d x \\
& \quad \leq c \int_{D}\left|X u-X u_{0}\right|^{p-\delta} d x+\frac{c}{R^{p-\delta}} \int_{2 B}^{\left|u-u_{0}\right|^{p-\delta} d x} \\
& \quad \leq c \int_{D}\left|X u-X u_{0}\right|^{p-\delta} d x+\frac{c|2 B|}{R^{p-\delta}}\left(\frac{1}{\operatorname{cap}_{p-\delta}\left(N\left(u-u_{0}\right), 2 B\right)} \int_{2 B}\left|X u-X u_{0}\right|^{p-\delta} d x\right),
\end{aligned}
$$

where $N\left(u-u_{0}\right)=\left\{x \in \bar{B}: u(x)=u_{0}(x)\right\}$. Since $u-u_{0}$ vanishes outside $\Omega$, we have $\mathbf{R}^{n} \backslash \Omega \subset$ $\left\{u-u_{0}=0\right\}$. On the other hand, by Lemma 2.7 and assumption $\left(H_{2}\right)$, there exists $\delta_{0}$ such that if $0<\delta<\delta_{0}, \mathbf{R}^{n} \backslash \Omega$ is uniformly $(X, p-\delta)$-fat, and hence

$$
\begin{aligned}
\operatorname{cap}_{p-\delta}\left(N\left(u-u_{0}\right), 2 B\right) & \geq \operatorname{cap}_{p-\delta}\left(\bar{B} \cap\left(\mathbf{R}^{n} \backslash \Omega\right), 2 B\right) \\
& \geq c \operatorname{cap}_{p-\delta}(\bar{B}, 2 B) \geq c|B| R^{-(p-\delta)} .
\end{aligned}
$$

From (3.6) and the doubling condition, we derive

$$
\begin{aligned}
c \int_{3 B \cap \Omega}(M|X \hat{u}|)^{p-\delta} d x & \leq c \int_{D}|X \hat{u}|^{p-\delta} d x \\
& \leq c \int_{D}|X u|^{p-\delta} d x+c \int_{D}\left|X u_{0}\right|^{p-\delta} d x,
\end{aligned}
$$

and then (3.5) becomes

$$
P \leq c \int_{3 B \cap \Omega}|u|^{p-\delta} d x+c \int_{3 B \cap \Omega}\left|X u_{0}\right|^{p-\delta} d x+c \int_{3 B \cap \Omega}|X u|^{p-\delta} d x .
$$

As regards the estimation of $L$, by changing the order of integration, we have

$$
\begin{aligned}
L= & \int_{\mu_{0}}^{\infty} \int_{D} \mu^{-(1+\delta)}(A(x, u, X u) \cdot X \hat{u}+B(x, u, X u) \hat{u}) \chi_{\{M|X \hat{u}|(x) \leq \mu\}} d x d \mu \\
= & \int_{D \backslash E_{\mu_{0}}} \int_{M|X \hat{u}|}^{\infty} \mu^{-(1+\delta)}(A(x, u, X u) \cdot X \hat{u}+B(x, u, X u) \hat{u}) d x d \mu \\
& +\int_{D \cap E_{\mu_{0}}} \int_{\mu_{0}}^{\infty} \mu^{-(1+\delta)}(A(x, u, X u) \cdot X \hat{u}+B(x, u, X u) \hat{u}) d x d \mu
\end{aligned}
$$




$$
\begin{aligned}
= & \frac{1}{\delta} \int_{D \backslash E_{\mu_{0}}}(M|X \hat{u}|)^{-\delta}(A(x, u, X u) \cdot X \hat{u}+B(x, u, X u) \hat{u}) d x \\
& +\frac{1}{\delta} \int_{D \cap E_{\mu_{0}}} \mu_{0}^{-\delta}(A(x, u, X u) \cdot X \hat{u}+B(x, u, X u) \hat{u}) d x
\end{aligned}
$$

Since $D \backslash E_{\mu_{0}}=D \backslash\left(D \cap E_{\mu_{0}}\right),(1.3)$ and (1.4) imply

$$
\begin{aligned}
L= & \frac{1}{\delta} \int_{D}(M|X \hat{u}|)^{-\delta} A(x, u, X u) \cdot X \hat{u} d x-\frac{1}{\delta} \int_{D \cap E_{\mu_{0}}}(M|X \hat{u}|)^{-\delta} A(x, u, X u) \cdot X \hat{u} d x \\
& +\frac{1}{\delta} \int_{D \backslash E_{\mu_{0}}}(M|X \hat{u}|)^{-\delta} B(x, u, X u) \hat{u} d x \\
& +\frac{1}{\delta} \int_{D \cap E_{\mu_{0}}} \mu_{0}^{-\delta}(A(x, u, X u) \cdot X \hat{u}+B(x, u, X u) \hat{u}) d x \\
\geq & \frac{1}{\delta} \int_{D}(M|X \hat{u}|)^{-\delta} A(x, u, X u) \cdot X \hat{u} d x \\
& -\frac{2 \alpha}{\delta} \int_{D \cap E_{\mu_{0}}}(M|X \hat{u}|)^{-\delta}\left(|u|^{p-1}+|X u|^{p-1}\right)|X \hat{u}| d x \\
& -\frac{\alpha}{\delta} \int_{D}(M|X \hat{u}|)^{-\delta}\left(|u|^{p-1}+|X u|^{p-1}\right)|\hat{u}| d x \\
:= & \frac{1}{\delta}\left(I_{1}-2 \alpha I_{2}-\alpha I_{3}\right) .
\end{aligned}
$$

Step 2. Next, we will estimate $I_{i}(i=1,2,3)$ one by one.

Now for estimation of $I_{1}$. To this end, define the sets

$$
\begin{aligned}
& D_{1}=\left\{x \in D \backslash B: M|X \hat{u}| \leq \delta\left(M_{D}\left|X u-X u_{0}\right|\right)\right\}, \\
& D_{2}=\left\{x \in D \backslash B: M|X \hat{u}|>\delta\left(M_{D}\left|X u-X u_{0}\right|\right)\right\}
\end{aligned}
$$

and $B_{\Omega}=B \cap \Omega$. Thus

$$
\begin{aligned}
I_{1}= & \int_{B_{\Omega} \cup D_{2}}(M|X \hat{u}|)^{-\delta}\left(A(x, u, X u)-A\left(x, u_{0}, X u_{0}\right)\right) \cdot \eta X\left(u-u_{0}\right) d x \\
& +\int_{B_{\Omega} \cup D_{2}}(M|X \hat{u}|)^{-\delta} A\left(x, u_{0}, X u_{0}\right) \cdot \eta\left(X u-X u_{0}\right) d x \\
& +\int_{D_{2}}(M|X \hat{u}|)^{-\delta} A(x, u, X u) \cdot X \eta\left(u-u_{0}\right) d x \\
& +\int_{D_{1}}(M|X \hat{u}|)^{-\delta} A(x, u, X u) \cdot X \hat{u} d x .
\end{aligned}
$$

Since $(M|X \hat{u}|)^{-\delta} \leq|X \hat{u}|^{-\delta}$ a.e., it follows from (1.5) and (1.3) that

$$
\begin{aligned}
I_{1} \geq & \beta \int_{B_{\Omega}}(M|X \hat{u}|)^{-\delta}\left|X u-X u_{0}\right|^{p} d x \\
& -\alpha\left(\int_{B_{\Omega}}|X \hat{u}|^{-\delta}\left(\left|u_{0}\right|^{p-1}+\left|X u_{0}\right|^{p-1}\right)\right. \\
& \times\left|X u-X u_{0}\right| d x+\int_{D_{2}}(M|X \hat{u}|)^{-\delta}\left(\left|u_{0}\right|^{p-1}+\left|X u_{0}\right|^{p-1}\right)\left|X u-X u_{0}\right| d x
\end{aligned}
$$




$$
\begin{aligned}
& \quad+\int_{D_{2}}(M|X \hat{u}|)^{-\delta}\left(|u|^{p-1}+|X u|^{p-1}\right)\left|X \eta\left(u-u_{0}\right)\right| d x \\
& \left.\quad+\int_{D_{1}}(M|X \hat{u}|)^{-\delta}\left(|u|^{p-1}+|X u|^{p-1}\right)|X \hat{u}| d x\right) \\
& :=I_{11}-\alpha\left(I_{12}+I_{13}+I_{14}+I_{15}\right) .
\end{aligned}
$$

Since the function $(M|X \hat{u}|)^{-\delta}$ is an $A_{p}$-weight, we obtain from Lemma 2.6 that

$$
I_{11} \geq c \beta \int_{B_{\Omega}}(M|X \hat{u}|)^{-\delta}\left(M_{B_{\Omega}}\left|X u-X u_{0}\right|\right)^{p} d x
$$

By the doubling condition and Lemma 2.8 we see that, for $x \in \frac{B}{2} \cap \Omega$,

$$
\begin{aligned}
M|X \hat{u}|(x) \leq & \sup _{B^{\prime} \ni x, B^{\prime} \subset B} f_{B^{\prime}}|X \hat{u}| d y+\sup _{B^{\prime} \ni x, B^{\prime} \cap \partial B \neq \emptyset} f_{B^{\prime}}|X \hat{u}| d y \\
\leq & M_{B_{\Omega}}\left|X\left(u-u_{0}\right)\right|+\frac{c}{R}\left(f_{2 B}\left|u-u_{0}\right|^{s^{\prime}} d x\right)^{\frac{1}{s^{\prime}}}+c\left(\frac{1}{|2 B|} \int_{D}\left|X u-X u_{0}\right|^{s^{\prime}} d x\right)^{\frac{1}{s^{\prime}}} \\
\leq & M_{B_{\Omega}}\left|X\left(u-u_{0}\right)\right|+\frac{c}{R}\left(\frac{1}{\operatorname{cap}_{s^{\prime}}\left(N\left(u-u_{0}\right), 2 B\right)} \int_{2 B}\left|X\left(u-u_{0}\right)\right|^{s^{\prime}} d x\right)^{\frac{1}{s^{\prime}}} \\
& +c\left(\frac{1}{|2 B|} \int_{D}\left|X u-X u_{0}\right|^{s^{\prime}} d x\right)^{\frac{1}{s^{\prime}}} \\
\leq & M_{B_{\Omega}}\left|X\left(u-u_{0}\right)\right|+c\left(\frac{1}{|2 B|} \int_{D}\left|X u-X u_{0}\right|^{s^{\prime}} d x\right)^{\frac{1}{s^{\prime}}}
\end{aligned}
$$

where $\max \left\{1,(p-\delta)_{*}\right\}<s^{\prime}<p-\delta$ is such that $\mathbf{R}^{n} \backslash \Omega$ is uniformly $\left(X, s^{\prime}\right)$-fat and the last inequality comes from an argument similar to (3.6).

To continue, we define

$$
G=\left\{x \in \frac{B}{2} \cap \Omega: M_{B_{\Omega}}\left|X\left(u-u_{0}\right)\right| \geq c\left(\frac{1}{|2 B|} \int_{D}\left|X u-X u_{0}\right|^{s^{\prime}} d x\right)^{\frac{1}{s^{\prime}}}\right\} .
$$

So from (3.11) we see that $M|X \hat{u}| \leq c M_{B_{\Omega}}\left|X\left(u-u_{0}\right)\right|$ on $G$, and then

$$
\begin{aligned}
I_{11} & \geq c \int_{G}\left(M_{B_{\Omega}}\left|X u-X u_{0}\right|\right)^{-\delta}\left(M_{B_{\Omega}}\left|X u-X u_{0}\right|\right)^{p} d x \\
& \geq c \int_{\frac{B}{2} \cap \Omega}\left|X u-X u_{0}\right|^{p-\delta} d x-c|B|\left(\frac{1}{|2 B|} \int_{D}\left|X u-X u_{0}\right|^{s^{\prime}} d x\right)^{\frac{p-\delta}{s^{\prime}}} \\
& \geq c \int_{\frac{B}{2} \cap \Omega}|X u|^{p-\delta} d x-c \int_{D}\left|X u_{0}\right|^{p-\delta} d x-c|B|\left(\frac{1}{|2 B|} \int_{D}|X u|^{s^{\prime}} d x\right)^{\frac{p-\delta}{s^{\prime}}} .
\end{aligned}
$$

Using the fact $X \hat{u}=X\left(u-u_{0}\right)$ on $B$ and Young's inequality, we have

$$
\begin{aligned}
I_{12} & \leq c \int_{D}\left(\left|u_{0}\right|^{p-\delta}+\left|X u_{0}\right|^{p-\delta}\right) d x+c \varepsilon \int_{D}\left|X u-X u_{0}\right|^{p-\delta} d x \\
& \leq c \int_{D}\left|u_{0}\right|^{p-\delta} d x+c \int_{D}\left|X u_{0}\right|^{p-\delta} d x+c \varepsilon \int_{D}|X u|^{p-\delta} d x .
\end{aligned}
$$


Next from the definition of $D_{2}$ and Lemma 2.4, we see

$$
\begin{aligned}
I_{13} & \leq c \delta^{-\delta} \int_{D}\left(M_{D}\left|X u-X u_{0}\right|\right)^{1-\delta}\left(\left|u_{0}\right|^{p-1}+\left|X u_{0}\right|^{p-1}\right) d x \\
& \leq c \int_{D}\left(\left|u_{0}\right|^{p-\delta}+\left|X u_{0}\right|^{p-\delta}\right) d x+c \varepsilon \int_{D}\left|X u-X u_{0}\right|^{p-\delta} d x \\
& \leq c \int_{D}\left|u_{0}\right|^{p-\delta} d x+c \int_{D}\left|X u_{0}\right|^{p-\delta} d x+c \varepsilon \int_{D}|X u|^{p-\delta} d x .
\end{aligned}
$$

For $I_{14}$, we have by using $\left|X \eta\left(u-u_{0}\right)\right| \leq|X \hat{u}|+\left|X u-X u_{0}\right|$

$$
\begin{aligned}
I_{14} \leq & c \int_{D_{2}}(M|X \hat{u}|)^{-\delta}\left(|u|^{p-1}+\left|X u-X u_{0}\right|^{p-1}+\left|X u_{0}\right|^{p-1}\right)\left|X \eta\left(u-u_{0}\right)\right| d x \\
\leq & c \int_{D_{2}}(M|X \hat{u}|)^{-\delta}\left(|u|^{p-1}+\left|X u_{0}\right|^{p-1}\right)\left(|X \hat{u}|+\left|X u-X u_{0}\right|\right) d x \\
& +c \int_{D_{2}}(M|X \hat{u}|)^{-\delta}\left|X u-X u_{0}\right|^{p-1}|X \eta|\left|u-u_{0}\right| d x \\
\leq & c \int_{D_{2}}(M|X \hat{u}|)^{-\delta}\left(|u|^{p-1}+\left|X u_{0}\right|^{p-1}\right)|X \hat{u}| d x \\
& +c \int_{D_{2}}(M|X \hat{u}|)^{-\delta}\left(|u|^{p-1}+\left|X u_{0}\right|^{p-1}\right)\left|X u-X u_{0}\right| d x \\
& +\frac{c}{R} \int_{D_{2}}(M|X \hat{u}|)^{-\delta}\left|X u-X u_{0}\right|^{p-1}\left|u-u_{0}\right| d x \\
:= & K_{1}+K_{2}+K_{3} .
\end{aligned}
$$

Using Young's inequality and (3.7), we get

$$
\begin{aligned}
K_{1} & \leq c \int_{D}\left(|u|^{p-1}+\left|X u_{0}\right|^{p-1}\right)|X \hat{u}|^{1-\delta} d x \\
& \leq c \int_{D}|u|^{p-\delta} d x+c \int_{D}\left|X u_{0}\right|^{p-\delta} d x+c \varepsilon \int_{D}|X u|^{p-\delta} d x .
\end{aligned}
$$

By the definition of $D_{2}$ and noting that $\left|X\left(u-u_{0}\right)\right| \leq M_{D}\left|X\left(u-u_{0}\right)\right|$ a.e. $D$,

$$
\begin{aligned}
K_{2} & \leq c \delta^{-\delta} \int_{D}\left|X u-X u_{0}\right|^{1-\delta}\left(|u|^{p-1}+\left|X u_{0}\right|^{p-1}\right) d x \\
& \leq c \int_{D}|u|^{p-\delta} d x+c \int_{D}\left|X u_{0}\right|^{p-\delta} d x+c \varepsilon \int_{D}|X u|^{p-\delta} d x .
\end{aligned}
$$

Finally, by Young's inequality,

$$
\begin{aligned}
K_{3} & \leq \frac{c \delta^{-\delta}}{R} \int_{D_{2}}\left(M_{D}\left|X u-X u_{0}\right|\right)^{-\delta}\left|X u-X u_{0}\right|^{p-1}\left|u-u_{0}\right| d x \\
& \leq \frac{c \delta^{-\delta}}{R} \int_{D}\left|X u-X u_{0}\right|^{p-1-\delta}\left|u-u_{0}\right| d x \\
& \leq c \varepsilon \int_{D}\left|X u-X u_{0}\right|^{p-\delta} d x+c \int_{D}\left|\frac{u-u_{0}}{R}\right|^{p-\delta} d x .
\end{aligned}
$$


Du and Han Boundary Value Problems ( 2017) 2017:93

Page 13 of 17

In order to estimate the second component of the right-hand side, we let $s^{\prime \prime}=(p-\delta)(1-\vartheta)$, where $0<\vartheta<\frac{p-\delta}{p-\delta+Q}$ if $p-\delta \leq Q$ and $0<\vartheta<\min \left\{\frac{p-\delta-Q}{p-\delta}, \frac{1}{2}\right\}$ if $p-\delta>Q$. Denote

$$
\kappa= \begin{cases}\frac{Q}{Q-s^{\prime \prime}}, & s^{\prime \prime}<Q, \\ 2, & s^{\prime \prime}>Q\end{cases}
$$

then $\kappa s^{\prime \prime} \geq p-\delta$. Using Lemma 2.7 and Lemma 2.8, we derive

$$
\begin{aligned}
\left(f_{2 B}\left|\frac{u-u_{0}}{R}\right|^{p-\delta} d x\right)^{\frac{1}{p-\delta}} & \leq c R^{-1}\left(f_{2 B}\left|u-u_{0}\right|^{\kappa s^{\prime \prime}} d x\right)^{\frac{1}{\kappa s^{\prime \prime}}} \\
& \leq c R^{-1}\left(\frac{1}{\operatorname{cap}_{s^{\prime \prime}}\left(N\left(u-u_{0}\right), 2 B\right)} \int_{2 B}\left|X\left(u-u_{0}\right)\right|^{s^{\prime \prime}} d x\right)^{\frac{1}{s^{\prime \prime}}} \\
& \leq c\left(\frac{1}{|2 B|} \int_{D}\left|X u-X u_{0}\right|^{s^{\prime \prime}}\right)^{\frac{1}{s^{\prime \prime}}}
\end{aligned}
$$

where the proof of the last inequality is similar to (3.6). Therefore,

$$
c \int_{2 B}\left|\frac{u-u_{0}}{R}\right|^{p-\delta} d x \leq c|2 B|\left(\frac{1}{|2 B|} \int_{D}\left|X u-X u_{0}\right|^{s^{\prime \prime}} d x\right)^{\frac{p-\delta}{s^{\prime \prime}}}
$$

Inserting (3.18) into (3.17), we have

$$
\begin{aligned}
K_{3} \leq & c \varepsilon \int_{D}|X u|^{p-\delta} d x+c \int_{D}\left|X u_{0}\right|^{p-\delta} d x \\
& +c|2 B|\left(\frac{1}{|2 B|} \int_{D}|X u|^{s^{\prime \prime}} d x\right)^{\frac{p-\delta}{s^{\prime \prime}}} .
\end{aligned}
$$

A combination of (3.15), (3.16) and (3.19) implies

$$
\begin{aligned}
I_{14} \leq & c \int_{D}\left(|u|^{p-\delta}+\left|X u_{0}\right|^{p-\delta}\right) d x \\
& +c \varepsilon \int_{D}|X u|^{p-\delta} d x+c|2 B|\left(\frac{1}{|2 B|} \int_{D}|X u|^{s^{\prime \prime}} d x\right)^{\frac{p-\delta}{s^{\prime \prime}}} .
\end{aligned}
$$

The definition of $D_{1}$ and Lemma 2.4 give

$$
\begin{aligned}
I_{15} & \leq c \int_{D_{1}}(M|X \hat{u}|)^{1-\delta}\left(|u|^{p-1}+|X u|^{p-1}\right) d x \\
& \leq c \delta^{1-\delta} \int_{D}\left(M_{D}\left|X u-X u_{0}\right|\right)^{1-\delta}\left(|u|^{p-1}+|X u|^{p-1}\right) d x \\
& \leq c \delta^{1-\delta}\left[\int_{D}\left|X u-X u_{0}\right|^{p-\delta} d x+\int_{D}\left(|u|^{p-\delta}+|X u|^{p-\delta}\right) d x\right] \\
& \leq c \int_{D}\left(|u|^{p-\delta}+\left|X u_{0}\right|^{p-\delta}\right) d x+c \delta \int_{D}|X u|^{p-\delta} d x .
\end{aligned}
$$


The previous estimates show that

$$
\begin{aligned}
I_{1} \geq & c \int_{\frac{B}{2} \cap \Omega}|X u|^{p-\delta} d x-c \int_{D}\left(|u|^{p-\delta}+\left|u_{0}\right|^{p-\delta}+\left|X u_{0}\right|^{p-\delta}\right) d x \\
& -c(\varepsilon+\delta) \int_{D}|X u|^{p-\delta} d x-c|2 B|\left(\frac{1}{|2 B|} \int_{D}|X u|^{t} d x\right)^{\frac{p-\delta}{t}},
\end{aligned}
$$

where $t=\max \left\{s^{\prime}, s^{\prime \prime}\right\}<p-\delta$.

Now we address the estimation of $I_{2}$. Using (3.7), we have

$$
\begin{aligned}
I_{2} \leq & \int_{D}|u|^{p-1}|X \hat{u}|^{1-\delta} d x+\int_{D \cap E_{\mu_{0}}}(M|X \hat{u}|)^{-\delta}|X u|^{p-1}|X \hat{u}| d x \\
\leq & c \int_{D}|u|^{p-\delta} d x+c \varepsilon \int_{D}|X \hat{u}|^{p-\delta} d x+\int_{D \cap E_{\mu_{0}}}|X u|^{p-1}(M|X \hat{u}|)^{1-\delta} d x \\
\leq & c \int_{D}\left(|u|^{p-\delta}+\left|X u_{0}\right|^{p-\delta}\right) d x \\
& +c \varepsilon \int_{D}|X u|^{p-\delta} d x+\int_{D \cap E_{\mu_{0}}}|X u|^{p-1}(M|X \hat{u}|)^{1-\delta} d x
\end{aligned}
$$

To estimate the last integral in (3.23), let $0<\tau<\frac{1}{2}$ and $x \in D \cap E_{\mu_{0}}$. If $|X u| \geq \tau^{-1} \mu_{0}$, then $M|X \hat{u}| \leq \mu_{0} \leq \tau|X u|$ and

$$
|X u|^{p-1}(M|X \hat{u}|)^{1-\delta} \leq|X u|^{p-1}(\tau|X u|)^{1-\delta}=\tau^{1-\delta}|X u|^{p-\delta}
$$

if $|X u|<\tau^{-1} \mu_{0}$, then

$$
|X u|^{p-1}(M|X \hat{u}|)^{1-\delta} \leq\left(\tau^{-1} \mu_{0}\right)^{p-1} \mu_{0}^{1-\delta} \leq \tau^{1-p} \mu_{0}^{p-\delta}
$$

By (3.24) and (3.25), we deduce that, for any $x \in D \cap E_{\mu_{0}}$,

$$
|X u|^{p-1}(M|X \hat{u}|)^{1-\delta} \leq c\left(\tau^{1-\delta}|X u|^{p-\delta}+\tau^{1-p} \mu_{0}^{p-\delta}\right) .
$$

For the second term in (3.26), we first observe from the proof of (3.11) that

$$
\frac{1}{R}\left(f_{2 B}\left|u-u_{0}\right|^{s^{\prime}} d x\right)^{\frac{1}{s^{\prime}}} \leq c\left(\frac{1}{|2 B|} \int_{D}\left|X u-X u_{0}\right|^{s^{\prime}} d x\right)^{\frac{1}{s^{\prime}}}
$$

Noticing $\mu_{0}=\frac{c}{|2 B|} \int_{D}|X \hat{u}| d x$, we have from Hölder's inequality

$$
\begin{aligned}
\tau^{1-p} \mu_{0}^{p-\delta} \leq & c \tau^{1-p}\left(\frac{1}{|2 B|} \int_{D}\left|X \eta\left(u-u_{0}\right)+\eta X\left(u-u_{0}\right)\right| d x\right)^{p-\delta} \\
\leq & c \tau^{1-p}\left(\frac{1}{R}\left(f_{2 B}\left|u-u_{0}\right|^{s^{\prime}} d x\right)^{\frac{1}{s^{\prime}}}\right)^{p-\delta} \\
& +c \tau^{1-p}\left(\frac{1}{|2 B|} \int_{D}\left|X\left(u-u_{0}\right)\right|^{s^{\prime}} d x\right)^{\frac{p-\delta}{s^{\prime}}}
\end{aligned}
$$




$$
\begin{aligned}
& \leq c \tau^{1-p}\left(\frac{1}{|2 B|} \int_{D}\left|X\left(u-u_{0}\right)\right|^{s^{\prime}} d x\right)^{\frac{p-\delta}{s^{\prime}}} \\
& \leq c \tau^{1-p}\left(\frac{1}{|2 B|} \int_{D}\left|X u_{0}\right|^{p-\delta} d x\right)+c \tau^{1-p}\left(\frac{1}{|2 B|} \int_{D}|X u|^{s^{\prime}} d x\right)^{\frac{p-\delta}{s^{\prime}}} .
\end{aligned}
$$

By (3.26) and (3.27), it follows that

$$
\begin{aligned}
& \int_{D \cap E_{\mu_{0}}}|X u|^{p-1}(M|X \hat{u}|)^{1-\delta} d x \\
& \quad \leq c \tau^{1-\delta} \int_{D}|X u|^{p-\delta} d x+c \int_{D}\left|X u_{0}\right|^{p-\delta} d x+c \tau^{1-p}|2 B|\left(\frac{1}{|2 B|} \int_{D}|X u|^{s^{\prime}} d x\right)^{\frac{p-\delta}{s^{\prime}}}
\end{aligned}
$$

Taking (3.28) into (3.23), we have

$$
\begin{aligned}
I_{2} \leq & c \int_{D}|u|^{p-\delta} d x+c \int_{D}\left|X u_{0}\right|^{p-\delta} d x \\
& +c\left(\varepsilon+\tau^{1-\delta}\right) \int_{D}|X u|^{p-\delta} d x+c \tau^{1-p}|2 B|\left(\frac{1}{|2 B|} \int_{D}|X u|^{s^{\prime}} d x\right)^{\frac{p-\delta}{s^{\prime}}} .
\end{aligned}
$$

For the estimation of $I_{3}$ : From (2.3), Lemma 2.8 and a similar process to the proof of (3.18), we have

$$
\begin{aligned}
I_{3} \leq & c \int_{D}\left(|u|^{p-1}+|X u|^{p-1}\right)|\hat{u}|^{1-\delta} d x \\
\leq & \int_{D}|u|^{p-\delta}+c \varepsilon \int_{D}|X u|^{p-\delta} d x+c \int_{D}\left|u-u_{0}\right|^{p-\delta} d x \\
\leq & c \int_{D}\left(|u|^{p-\delta}+\left|X u_{0}\right|^{p-\delta}\right) d x \\
& +c \varepsilon \int_{D}|X u|^{p-\delta} d x+c|2 B|\left(\frac{1}{|2 B|} \int_{D}|X u|^{t} d x\right)^{\frac{p-\delta}{t}}
\end{aligned}
$$

where $t=\max \left\{s^{\prime}, s^{\prime \prime}\right\}<p-\delta$.

Step 3. Taking into account (3.4), (3.8), substituting (3.22), (3.29) and (3.30) into (3.9), and letting $\varepsilon=\tau^{1-\delta}$, it follows that

$$
\begin{aligned}
& \int_{\frac{B}{2} \cap \Omega}|X u|^{p-\delta} d x \\
& \quad \leq c \int_{3 B \cap \Omega}\left(|u|^{p-\delta}+\left|u_{0}\right|^{p-\delta}+\left|X u_{0}\right|^{p-\delta}\right) d x \\
& \quad+c\left(\delta+\tau^{1-\delta}\right) \int_{3 B \cap \Omega}|X u|^{p-\delta} d x+c \tau^{1-p}|2 B|\left(\frac{1}{|2 B|} \int_{3 B \cap \Omega}|X u|^{t} d x\right)^{\frac{p-\delta}{t}} .
\end{aligned}
$$

To sum up the cases $3 B \subset \Omega$ and $3 B \backslash \Omega \neq \emptyset$, we let

$$
g(x)= \begin{cases}|X u|^{t}, & x \in \Omega, \\ 0, & x \in \mathbf{R}^{n} \backslash \Omega,\end{cases}
$$


and

$$
f(x)= \begin{cases}\left(\left|u-u_{0}\right|+\left|u_{0}\right|+\left|X u_{0}\right|\right)^{t}, & x \in \Omega \\ 0, & x \in \mathbf{R}^{n} \backslash \Omega\end{cases}
$$

Thus we have from (3.1) and (3.31)

$$
f_{\frac{B}{2}} g^{q} d x \leq b\left[\left(f_{3 B} g d x\right)^{q}+f_{3 B} f^{q} d x\right]+\theta f_{3 B} g^{q} d x
$$

where $q=\frac{p-\delta}{t}, \theta=c\left(\delta+\tau^{1-\delta}\right)$ and $b=c \tau^{1-p}$. Choosing $\tau, \delta$ small enough, we see by Lemma 2.3 that there exists $t_{1}=p-\delta+\varepsilon_{0}$, for some $\varepsilon_{0}>0$, such that $|X u| \in L^{t_{1}}(\Omega)$.

Furthermore, we will show that there exists $t_{2}>r=p-\delta$ such that $u \in L^{t_{2}}(\Omega)$. Since $u-u_{0} \in W_{X, 0}^{1, r}(\Omega)$, we obtain from Lemma 2.2 that, for $r<Q, r^{*}=Q r /(Q-r)$,

$$
\left(\int_{\Omega}\left|u-u_{0}\right|^{r^{*}} d x\right)^{\frac{1}{r^{*}}} \leq C(\Omega)\left(\int_{\Omega}\left|X\left(u-u_{0}\right)\right|^{r} d x\right)^{\frac{1}{r}}<\infty .
$$

Taking $t_{2}=\min \left\{s, r^{*}\right\}>r$, we have

$$
\begin{aligned}
\left(\int_{\Omega}|u|^{t_{2}} d x\right)^{\frac{1}{t_{2}}} & \leq\left(\int_{\Omega}\left|u-u_{0}\right|^{t_{2}} d x\right)^{\frac{1}{t_{2}}}+\left(\int_{\Omega}\left|u_{0}\right|^{t_{2}} d x\right)^{\frac{1}{t_{2}}} \\
& \leq C\left(\int_{\Omega}\left|u-u_{0}\right|^{r^{*}} d x\right)^{\frac{1}{r^{*}}}+\left(\int_{\Omega}\left|u_{0}\right|^{t_{2}} d x\right)^{\frac{1}{t_{2}}}
\end{aligned}
$$

and then $u \in L^{t_{2}}(\Omega)$ by $u_{0} \in L^{s}(\Omega)$. If $r \geq Q$ then we can apply the above reasoning for any $r^{*}<\infty$ to obtain $u \in L^{t_{2}}(\Omega)$.

We set $\tilde{p}=\min \left\{t_{1}, t_{2}\right\}>p-\delta$ and $u \in W_{X}^{1, \tilde{p}}(\Omega)$. Repeating the preceding reasoning, we know that there exists $\tilde{\delta}>0$ such that $u \in W_{X}^{1, p+\tilde{\delta}}(\Omega)$ and the proof is complete.

\section{Conclusions}

In this paper, we obtained the global higher integrability for very weak solutions to the Dirichlet problem for a nonlinear subelliptic equation on Carnot-Carathéodory spaces which implies that such solutions are classical weak solutions. It is a generalization of the corresponding result in the classical Euclidean setting.

\section{Acknowledgements}

The authors are grateful to anonymous reviewers for their careful reading of this paper and their insightful comments and suggestions, which improved the paper a lot. The current work is supported by the National Natural Science Foundation of China (No. 11271299).

Competing interests

The authors declare that they have no competing interests.

Authors' contributions

All authors read and approved the final manuscript.

Publisher's Note

Springer Nature remains neutral with regard to jurisdictional claims in published maps and institutional affiliations. 


\section{References}

1. Iwaniec, T, Sbordone, C: Weak minima of variational integrals. J. Reine Angew. Math. 454, 143-161 (1994)

2. Lewis, JL: On very weak solutions of certain elliptic systems. Commun. Partial Differ. Equ. 18(9-10), 1515-1537 (1993)

3. Giannetti, F, Passarelli di Napoli, A: On very weak solutions of degenerate $p$-harmonic equations. Nonlinear Differ. Equ. Appl. 14(5-6), 739-751 (2007)

4. Kinnunen, J, Lewis, JL: Very weak solutions of parabolic systems of p-Laplacian type. Ark. Mat. 40(1), 105-132 (2002)

5. Xie, S, Fang, A: Global higher integrability for the gradient of very weak solutions of a class of nonlinear elliptic systems. Nonlinear Anal. 53(7-8), 1127-1147 (2003)

6. Fattorusso, L, Molica Bisci, G, Tarsia, A: A global regularity result for some degenerate elliptic systems. Nonlinear Anal. $125,54-66(2015)$

7. Zatorska-Goldstein, A: Very weak solutions of nonlinear subelliptic equations. Ann. Acad. Sci. Fenn., Math. 30(2), 407-436 (2005)

8. Kilpeläinen, T, Koskela, P: Global integrability of the gradients of solutions to partial differential equations. Nonlinear Anal. 23(7), 899-909 (1994)

9. Danielli, D, Garofalo, N, Phuc, NC: Inequalities of Hardy-Sobolev type in Carnot-Carathéodory spaces. In: Sobolev Spaces in Mathematics. I: Sobolev Type Inequalities, pp. 117-151. Springer, New York (2009)

10. Hörmander, L: Hypoelliptic second order differential equations. Acta Math. 119, 147-171 (1967)

11. Chow, WL: Über systeme von linearen partiellen differentialgleichungen erster ordnung. Math. Ann. 117, 98-105 (1939)

12. Nagel, A, Stein, EM, Wainger, S: Balls and metrics defined by vector fields. I: basic properties. Acta Math. 155, 103-147 (1985)

13. Garofalo, N, Nhieu, DM: Lipschitz continuity, global smooth approximations and extension theorems for Sobolev functions in Carnot-Carathéodory spaces. J. Anal. Math. 74, 67-97 (1998)

14. Hajłasz, P, Koskela, P: Sobolev met Poincaré. Mem. Am. Math. Soc. 688, 1-101 (2000)

15. Lu, G: Weighted Poincaré and Sobolev inequalities for vector fields satisfying Hörmander's condition and applications. Rev. Mat. Iberoam. 8(3), 367-439 (1992)

16. Danielli, D: Regularity at the boundary for solutions of nonlinear subelliptic equations. Indiana Univ. Math. J. 44(1), 269-286 (1995)

17. Federer, H: Geometric Measure Theory. Die Grundlehren der Mathematischen Wissenschaften, vol. 153. Springer New York (1969)

\section{Submit your manuscript to a SpringerOpen ${ }^{\circ}$ journal and benefit from:}

- Convenient online submission

- Rigorous peer review

- Open access: articles freely available online

- High visibility within the field

- Retaining the copyright to your article 